RA and in biologic-treated patients than in controls $(p=0.005)$. On brain MRI scans, there were significantly more vascular lesions both in the left and the right side in RA patients $(55,1 \%>53,1 \%)$ than in controls $(23,5 \%>20,1 \%)(p<0,05)$, the cerebral atrophy is much more common in $\mathrm{RA}(0,26 \mathrm{vs.} 0,03 ; p=0,005)$.

Conclusions: These findings suggest that the presence of neuropsychiatric manifestations and cognitive impairment in RA patients is significant. Biologictreated patients may represent a more severe RA subset thus having cognitive dysfunction more commonly. Brain atrophy, emollition and vascular lesions are more often in RA patients than controls.

Disclosure of Interest: None declared

DOI: 10.1136/annrheumdis-2018-eular.2153

\section{FRI0090 ASSOCIATION OF BODY COMPOSITION WITH DISEASE ACTIVITY AND DISABILITY IN RHEUMATOID ARTHRITIS}

K.M. Son ${ }^{1}$, S.Y. Lee ${ }^{2}$, S.H. Kang ${ }^{2}$, Y.I. Seo ${ }^{2}$, H.A. Kim². ${ }^{1}$ Hallym University Dongtan Sacred Heart Hospital; ${ }^{2}$ Hallym University Sacred Heart Hospital, Gyeonggi-Do, Korea, Republic Of

Background: Rheumatoid arthritis (RA) is a chronic form of inflammatory arthritis characterised by multiple joint involvement and significant disability. Previous studies showed that RA is associated with considerable changes in body composition, lipid profile, adipokines and insulin sensitivity

Objectives: To explore the association of body composition with pain, disease activity and disability in rheumatoid arthritis (RA)

Methods: Three hundred thirty five patients with RA visiting the Hallym University Sacred Hospital underwent body composition measurement with inbody analyzer and examined the disease activity score (DAS28). The association of body mass index (BMI), body fat mass and skeletal muscle mass with DAS28, DAS28-P (an index derived to measure subjective component of DAS28), pain VAS and disability measured with the health assessment questionnaire (HAQ) was explored Obesity was defined as BMI $\geq 25 \mathrm{~kg} / \mathrm{m}^{2}$. Pain VAS was dichotomized as $\leq 40$ and $>40$. Low $H A Q$ score was defined as $\leq 0.5$. Logistic regression was divided in female versus male.

Results: Mean age of patients was $56 \pm 11.9$ years and $84.8 \%$ were female. The median(IQR) disease durations was 6 (3.5-9) years and mean DAS28 score was $3.55 \pm 1.14$. Mean BMI was $23.6 \pm 3.7 \mathrm{~kg} / \mathrm{m}^{2}$ and 109 patients $(32.5 \%)$ were obese Obese patients had higher CRP level (1.68 ml/dL vs $0 \mathrm{ml} / \mathrm{dL}, \mathrm{p}=0.013)$, ESR level ( $25 \mathrm{~mm} / \mathrm{hr}$ vs $18 \mathrm{~mm} / \mathrm{hr}, \mathrm{p}=0.032$ ), pain VAS score (40 vs $35, \mathrm{p}=0.045$ ), and higher DAS28-ESR score $(3.75 \pm 1.18$ vs $3.46 \pm 1.11, p=0.031)$, than non obese patients. In multivariable regression analysis, DAS28 score in female was positively associated with current steroid dose, HAQ and body fat mass. In univariable logistic regression, higher pain VAS category in female was associated with older age, higher BMI and higher body fat mass. In multivariable logistic regression analysis, higher HAQ score in female was associated with older age, higher DAS28, higher body fat/skeletal muscle ration and lower skeletal muscle mass. In multivariable regression analysis, DAS28-P score in female was positively associated with higher body fat/skeletal muscle ratio and negatively associated with positivity of anti-CCP

Conclusions: Body compositions such as body fat mass and skeletal muscle mass are significantly associated with pain and disability in RA patients

Disclosure of Interest: None declared

DOI: 10.1136/annrheumdis-2018-eular.2382

\section{\begin{tabular}{|l|l}
\hline FRI0091 SOMATOSENSORY DYSFUNCTION IN RHEUMATOID \\
\hline
\end{tabular} ARTHRITIS - A QUANTITATIVE SENSORY TESTING ASSESSMENT}

T.M. Rocha ${ }^{1}$, M. Barbosa ${ }^{2}$, S. Pimenta ${ }^{1}$, M. Bernardes ${ }^{1}$, A. Bernardo ${ }^{1}$, R. Lucas ${ }^{3}$, L. Costa ${ }^{1}$, J. Vollert ${ }^{4}$, C. Maier ${ }^{4}{ }^{1}$ Rheumatology, ${ }^{2}$ Pain Unit, Centro Hospitalar de São João; ${ }^{3}$ Clinical Epidemiology, Faculty of Medicine University of Porto, Porto, Portugal; ${ }^{4}$ Pain Management, BG-University Hospital Bergmannsheil GmbH, Bochum, Germany

Background: Significant pain persists in a substantial proportion of Rheumatoid Arthritis (RA) patients and features suggestive of neuropathic pain (NP) were described. Few studies applied quantitative sensory testing (QST) to evaluate the somatosensory phenotype of RA pain

Objectives: To explore the sensory abnormalities in RA and study its association with clinical and disease activity parameters

Methods: Cross-sectional study was performed with RA patients followed at our rheumatology department. QST was performed in patients classified with NP (according to LANSS and/or pain DETECT scores) in both the most painful and non-painful contralateral joint areas. The evaluation followed the protocol of the German Research Network on Neuropathic Pain. Patients with diagnosed neuropathy or non-RA risk factors for NP were excluded. Proportions of abnormal detection/pain thresholds were calculated after z-transformation of QST data based on gender, age and site reference values. Correlations were studied (Spearman correlation coefficient) and comparison between groups was performed (Mann-Whitney and $\chi^{2}$ tests)

Results: From 112 evaluated RA patients, 47\% were classified with NP and 39 performed QST. Thirty four (87\%) were women, with a mean age of $53.5 \pm 11.8$ years and median disease duration of 11 years $;{ }^{2-31} 74 \%$ were seropositive for Rheumatoid Factor and/or ACPA; 90\% were treated with conventional synthetic Disease-Modifying Antirheumatic Drugs (DMARDs) and 39\% with biological DMARDs (bDMARDs). Mean DAS28 CRP was 3.44 \pm 0.7 . For non-nociceptive parameters, $23(59 \%)$ patients exhibited sensory loss (Lo), 6 to thermal stimul (L1), 10 to mechanical stimuli (L2) and the reminder for both (L3). Concerning nociceptive parameters, hyperalgesia $(\mathrm{Ga})$ was noted in almost all the patients (97\%), 1 to thermal (G1), 20 to mechanical (G2) and 17 for both stimuli (G3). Twenty two (60\%) patients presented both Lo and Ga findings. Higher proportion of Lo was noted in bDMARDs group ( $86 \%$ vs $46 \%, p=0.02)$. Lo patients had significant lower median CRP and ESR levels, but no differences were observed concerning disease activity scores. Thermal Lo (L1 and L3) was also more frequent in the bDMARDs group (57\% vs $21 \%, \mathrm{p}=0.04)$ and cold Lo in hydroxychloroquine (HCQ) treated patients $(90 \%$ vs $21 \% \mathrm{p}=0.02)$. Cold Ga was more frequent in patients under methotrexate (MTX) $(48 \%$ vs $6 \%, p=0.04)$ and less frequent in the bDMARDs group ( $7 \%$ vs $46 \%, p<0.05$ ). A weak correlation of $Z$ cold detection and $Z$ warm detection values with CRP and ESR levels was noted $(r=0.34$ and $r=0.35$ $p=0.04)$. Time exposure to $H C Q, M T X$ and bDMARDs was negatively correlated with $Z$ cold detection $(r=-0.34, p=0.03)$, $Z$ pressure pain $(r=-0.33, p=0.04)$ and $Z$ vibration detection $(r=-0.32, p=0.04)$, respectively.

Conclusions: Almost all patients presented hyperalgesia, but a sizable proportion also had sensory loss, frequently involving A $\beta$ fibres. CRP and ESR levels possibly influence small fibre function, but no association with disease activity scores was found. Possible association of bDMARDs and HCQ treatment with sensory detection loss and of MTX with lower pain thresholds was pointed.

\section{REFERENCES:}

[1] Koop SM, et al. Arthritis Res Ther 2015;17:237

[2] Lee YC, et al. Arthritis Care Res (Hoboken). 2018;70(2):197-204.

Disclosure of Interest: T. Rocha Grant/research support from: Portuguese Society of Rheumatology/Alfa Wassermann on May 2015, M. Barbosa: None declared, S. Pimenta: None declared, M. Bernardes: None declared, A. Bernardo: None declared, R. Lucas: None declared, L. Costa: None declared, J. Vollert: None declared, C. Maier: None declared DOI: 10.1136/annrheumdis-2018-eular.5558

\section{FRI0092 THE ASSOCIATION OF PSYCHOLOGICAL STRESS WITH INFLAMMATION IN PATIENTS WITH CLINICALLY SUSPECT ARTHRALGIA - A STUDY DURING RHEUMATOID ARTHRITIS DEVELOPMENT}

A.C. Boer ${ }^{1}$, R.M. ten Brinck ${ }^{1}$, A.W.M. Evers ${ }^{2}$, A.H.M. van Der Helm - van Mil ${ }^{1,3}$ ${ }^{1}$ Rheumatology, Leiden University Medical Centre; ${ }^{2}$ Medical and Neuropsychology, Faculty of Social and Behavioural Science, Leiden University and Department of Psychiatry, Leiden University Medical Centre, Leiden; ${ }^{3}$ Rheumatology, Erasmus Medical Centre, Rotterdam, Netherlands

Background: Within established Rheumatoid Arthritis (RA), stress can have proinflammatory effects by activating the immune system via the hypothalamic-pituitary-adrenal axis and the autonomic nervous system. It is unknown if stress-levels promote also inflammation during RA-development.

Objectives: We studied whether the psychological stress response was increased in Clinically Suspect Arthralgia (CSA) and if this associated with inflam mation at presentation with arthralgia and with progression to clinical arthritis.

Methods: In 241 CSA-patients, psychological stress was measured by the Mental Health Inventory (MHI-5) and the Perceived Stress Scale (PSS-10) at first presentation and during follow-up. Systemic inflammation was measured by $\mathrm{C}$ reactive protein (CRP) and joint inflammation by $1.5 \mathrm{~T}-\mathrm{MRI}$ of wrist-, $\mathrm{MCP}$ - and MTP-joints.

Results: At baseline, $10 \%$ of CSA-patients had a high psychological stress response according to the $\mathrm{MHI}-5$. This was not different for patients presenting with or without an elevated CRP, with or without subclinical MRI-detected inflammation and for patients who did or did not develop arthritis. Similar findings were obtained with the PSS-10. When developing clinical arthritis, the percentage of patients with 'high psychological stress' increased to $31 \%(p=0.025)$; during the first year of treatment this decreased to $8 \%(p=0.020)$. 'High psychological stress' in non-progressors remained infrequent over time (range $7 \%-13 \%$ ). Stress was associated with fatigue $(p=0.003)$ and wellbeing $(p<0.001)$ 
Conclusions: Psychological stress was not increased in the phase of arthralgia, raised at the time of diagnoses and decreased thereafter. This temporal-relationship, and the lack of association with inflammation in arthralgia, argue against psychological stress having a significant contribution to inflammatory arthritis development.

Disclosure of Interest: None declared

DOI: 10.1136/annrheumdis-2018-eular.3278

\section{FRI0093 DON'T MISS THE DEPRESSION! COMORBIDITIES IN PATIENTS WITH RHEUMATOID ARTHRITIS AND THEIR IMPACT ON PATIENT-REPORTED OUTCOMES: RESULTS OF CLAIMS DATA LINKED TO A QUESTIONNAIRE SURVEY}

K. Albrecht ${ }^{1}$, A. Luque Ramos ${ }^{2}$, J. Callhoff ${ }^{1}$, I. Redeker ${ }^{1}$, F. Hoffmann ${ }^{2}$, A. Zink ${ }^{1}$. ${ }^{1}$ Epidemiology, German Rheumatism Research Center, Berlin; ${ }^{2}$ Health Services Research, Carl von Ossietzky University, Oldenburg, Germany

Background: Comorbidities are increasingly acknowledged as important clinical manifestation in patients with rheumatoid arthritis (RA)

Objectives: To investigate the prevalence of comorbidities in RA and their association with patient-reported outcomes.

Methods: Data of 96921 persons with RA and 484605 age- and sex-matched controls of a German statutory health fund were studied on the presence of selected comorbidities in 2015. Diagnoses and therapies were assigned to the provider (general practitioner, rheumatologist or another specialist). A selfreported questionnaire, comprising joint counts (TJC, SJC), functional status $(\mathrm{FFbH})$, impact of the disease (RAID) and well-being (WHO-5) was sent to a random sample of 6195 persons with RA of whom 3184 responded. For respondents who confirmed their RA $(n=2,535)$, the association between comorbidities and patient-reported outcomes were analysed by multivariable linear regression analyses.

Results: Compared to controls, persons with RA (mean age 63 years, $80 \%$ female) had higher prevalences of all comorbidities, the most common were depression and osteoporosis besides cardiovascular risk factors (table 1). The diagnosis of depression was provided in $50 \%$ of cases by general practitioners, in $13 \%$ by rheumatologists and in $48 \%$ by other specialists while the diagnosis of osteoporosis was made in $76 \%$ by general practitioners, in $48 \%$ by rheumatologists and in $46 \%$ by other specialists. Among the survey respondents, increasing numbers of comorbidities were associated with worse TJC, SJC, function and WHO- 5 values. Depression, obesity and osteoporosis had the highest impact on functional status and TJCs. The percentage of patients in rheumatologic care decreased from $73 \%$ with $0-1$ comorbidity to $62 \%$ with $\geq 8$ comorbidities (ageadjusted).

Abstract FRI0093 - Table 1. Prevalence of selected comorbidities in cases and controls

\begin{tabular}{lcc}
\hline & $\begin{array}{c}\text { RA patients } \mathrm{n}=96921 \\
\%(95 \% \mathrm{Cl})\end{array}$ & $\begin{array}{c}\text { Controls } \mathrm{n}=484605 \\
\%(95 \% \mathrm{Cl})\end{array}$ \\
\hline $\begin{array}{l}\text { Cardiovascular risk factors } \\
\text { Hypertension }\end{array}$ & $61.8(61.5-62.1)$ & $48.0(47.8-48.1)$ \\
Hyperlipidemia & $39.4(39.1-39.7)$ & $32.4(32.2-32.5)$ \\
Obesity & $17.7(17.5-18.0)$ & $12.3(12.2-12.3)$ \\
Comorbid disorders & & \\
Depression & $31.4(31.1-31.7)$ & $20.4(20.3-20.5)$ \\
Osteoporosis & $25.7(25.4-26.0)$ & $9.7(9.6-9.8)$ \\
Diabetes & $21.5(21.2-21.7)$ & $15.3(15.2-15.4)$ \\
Cardiac arrhythmia & $18.8(18.5-19.0)$ & $13.2(13.1-13.3)$ \\
Hypothyroidism & $17.9(17.6-18.1)$ & $12.8(12.7-12.9)$ \\
Coronary heart disease & $15.2(15.0-15.4)$ & $9.6(9.5-9.7)$ \\
Liver disease & $13.8(13.6-14.0)$ & $9.1(9.0-9.1)$ \\
Vascular diseases & $12.9(12.7-13.1)$ & $8.5(8.4-8.6)$ \\
Asthma & $12.3(12.1-12.5)$ & $7.4(7.3-7.4)$ \\
COPD & $11.3(11.0-11.4)$ & $6.6(6.5-6.6)$ \\
\hline
\end{tabular}

Conclusions: Osteoporosis and depression are amongst the most common disorders in persons with RA. While osteoporosis is usually taken into account by the rheumatologist, more attention should be paid to depression as both disorders strongly affect patient-reported outcomes.

Acknowledgements: This study was funded by the German Federal Ministry of Education and Research (01EC1405).

Disclosure of Interest: None declared

DOI: 10.1136/annrheumdis-2018-eular.2399

\section{FRI0094}

DOES SUBCLINICAL INFLAMMATION EXPLAIN JOINT PAIN IN PATIENTS WITH ARTHRALGIA SUSPICIOUS FOR PROGRESSION TO RHEUMATOID ARTHRITIS? RESULTS OF A CROSS-SECTIONAL MRI-STUDY

L.E. Burgers, R.M. ten Brinck, D.M. Boeters, A.H.M. van der Helm-van Mil. Rheumatology, Leiden University Medical Centre, Leiden, Netherlands

Background: The development of Rheumatoid Arthritis (RA) is often preceded by a symptomatic phase of arthralgia. The etiology of symptoms in this phase is unclear.

Objectives: Since subclinical joint inflammation is expected to be causally related to pain, we aimed to determine associations between subclinical MRI-detected inflammation and pain in patients with arthralgia suspicious for progression to RA. Methods: Unilateral MRIs of the wrist, MCP(2-5)- and MTP(1-5)-joints of 325 patients who fulfilled the EULAR definition of arthralgia suspicious for progression to RA were made and scored by two readers on subclinical inflammation (synovitis, bone marrow oedema (BME) and tenosynovitis). Associations between MRIdetected inflammation and overall pain severity at patient level (measured using the visual analogue scale (VAS)), and local tenderness at joint palpation, were studied in all patients, in ACPA-positive and ACPA-negative patients separately, and in the subgroup of patients that progressed to inflammatory arthritis

Results: At patient level, synovitis $(\beta=0.10, p=0.048)$ and tenosynovitis $(\beta=0.11$, $p=0.026)$ associated with the VAS-pain. Of the 1620 imaged joints, $447(28 \%)$ were tender. Subclinical inflammation was present in $32 \%$ of tender joints and in $25 \%$ of non-tender joints. MRI-detected synovitis associated independently with joint tenderness (OR 1.74, $\mathrm{p}<0.001$ ). In ACPA-negative patients synovitis associated independently with joint tenderness (OR 1.96, $\mathrm{p}<0.001$ ), while BME was independently associated with joint tenderness in ACPA-positive patients (OR $2.39, p=0.005)$. Sensitivity analyses in patients who developed arthritis during follow-up ( $n=61$ ) revealed similar associations.

Conclusions: In patients with arthralgia suspicious for progression to RA, joint tenderness and pain are associated with MRI-detected subclinical inflammation. The association is incomplete, indicating that subclinical inflammation is not the sole explanation of the arthralgia.

Disclosure of Interest: None declared

DOI: 10.1136/annrheumdis-2018-eular.3294

\section{FRI0095 ANTI-TNFA VERSUS RITUXIMAB IN REFRACTORY PERIPHERAL ULCERATIVE KERATITIS ASSOCIATED TO RHEUMATIC DISEASES. MULTICENTER STUDY OF 24 PATIENTS}

L.C. Domínguez Casas ${ }^{1}$, V. Calvo-Río ${ }^{1}$, O. Maíz-Alonso ${ }^{2}$, A. Blanco ${ }^{3}$, E. Beltran ${ }^{4}$, L. Martinez Acosta ${ }^{5}$, M.C. Alvarez de Buergo ${ }^{6}$, E. Rubio-Romero ${ }^{7}$, D. Díaz-Valle ${ }^{8}$, R. López-González ${ }^{9}$, A. García- Aparicio ${ }^{10}$, A. Juan Mas ${ }^{11}$, E. Pons ${ }^{1}$, R. DemetrioPablo ${ }^{12}$, N. Vegas-Revenga ${ }^{1}$, R. Blanco ${ }^{1}$, M. Gonzalez-Gay ${ }^{1} .{ }^{1}$ Rheumatology, HUMV, Santander, ${ }^{2}$ Rheumatology, ${ }^{3}$ Ophtalmology, Hospital Universitario Donostia, San Sebastian; ${ }^{4}$ Rheumatology, Hospital General Universitario de Valencia; ${ }^{5}$ Ophtalmology, Hospital Peset, Valencia; ${ }^{6}$ Rheumatology, Hospital Río Carrión, Palencia; ${ }^{7}$ Ophtalmology, Hospital Universitario Virgen del Rocío, Sevilla; ${ }^{8}$ Ophtalmology, Hospital Clínico San Carlos, Madrid; ${ }^{9}$ Rheumatology, Complejo hospitalario de Zamora, Zamora; ${ }^{10}$ Rheumatology, Hospital de Toledo, Toledo;

${ }^{11}$ Rheumatology, Hospital Son Laàtzer, Palma de Mallorca; ${ }^{12}$ Ophtalmology, HUMV, Santander, Spain

Background: This study shows that treatment with biologic drugs, including ant TNF drugs, in NION associated to IMIDs, refractory to conventional treatment, seems to be effective. These results must be confirmed in prospective and randomised trials.

Objectives: Our aim was to compare anti-TNF $\alpha$ vs Rituximab (RTX) in refractory PUK.

Methods: Multicenter study of 24 patients with PUK. All of them presented inadequate response to corticosteroids and at least 1 systemic traditional immunosuppressive drug.

Anti-TNF $\alpha$ were used in 17 patients: Adalimumab ( $\mathrm{n}=9) 40 \mathrm{mg} / \mathrm{sc}$ every 1-2 weeks, infliximab (IFX) ( $\mathrm{n}=7) 3-5 \mathrm{mg} / \mathrm{kg}$ iv $/ 4-6$ weeks, etanercept $(n=1) 50 \mathrm{mg} /$ week. RTX was used in 7 patients 1-2 g i.v. every 6 or 12 months.

The main outcomes were Best Corrected Visual Acuity (BCVA), signs of inflammation (scleritis and episcleritis), progression to corneal thinning, central keratolysis and ocular perforation. 